\title{
A Local Analysis of Imprimitive Symmetric Graphs
}

\author{
SANMING ZHOU* \\ smzhou@ms.unimelb.edu.au \\ Department of Mathematics and Statistics, The University of Melbourne, Parkville, VIC 3010, Australia \\ Received March 3, 2004; Revised April 13, 2005; Accepted May 12, 2005

\begin{abstract}
Let $\Gamma$ be a $G$-symmetric graph admitting a nontrivial $G$-invariant partition $\mathcal{B}$. Let $\Gamma_{\mathcal{B}}$ be the quotient graph of $\Gamma$ with respect to $\mathcal{B}$. For each block $B \in \mathcal{B}$, the setwise stabiliser $G_{B}$ of $B$ in $G$ induces natural actions on $B$ and on the neighbourhood $\Gamma_{\mathcal{B}}(B)$ of $B$ in $\Gamma_{\mathcal{B}}$. Let $G_{(B)}$ and $G_{[B]}$ be respectively the kernels of these actions. In this paper we study certain "local actions" induced by $G_{(B)}$ and $G_{[B]}$, such as the action of $G_{[B]}$ on $B$ and the
\end{abstract} \\ action of $G_{(B)}$ on $\Gamma_{\mathcal{B}}(B)$, and their influence on the structure of $\Gamma$.
}

Keywords: symmetric graph, arc-transitive graph, quotient graph, locally quasiprimitive graph

\section{Introduction}

Let $G$ be a finite group acting on a finite set $\Omega$. A partition $\mathcal{B}$ of $\Omega$ is $G$-invariant if $B^{g} \in \mathcal{B}$ for $B \in \mathcal{B}$ and $g \in G$, where $B^{g}:=\left\{\alpha^{g}: \alpha \in B\right\}$; and $\mathcal{B}$ is nontrivial if $1<|B|<|\Omega|$. If $\Omega$ admits a nontrivial $G$-invariant partition, then $G$ is said to be imprimitive on $\Omega$; otherwise $G$ is said to be primitive on $\Omega$. The group $G$ is regular on $\Omega$ if $G$ is transitive on $\Omega$ and the only element of $G$ that fixes a point of $\Omega$ is the identity. The kernel of the action of $G$ on $\Omega$ is defined to be the subgroup of all elements of $G$ which fix each point of $\Omega$. If this kernel is equal to the identity subgroup of $G$, then $G$ is said to be faithful on $\Omega$.

Let $\Gamma=(V(\Gamma), E(\Gamma))$ be a finite graph and $G$ a finite group. If $G$ acts on the vertex set $V(\Gamma)$ of $\Gamma$ such that $G$ preserves the adjacency of $\Gamma$, then $\Gamma$ is said to admit $G$ as a group of automorphisms. If such a group $G$ is transitive on $V(\Gamma)$ and, in its induced action, transitive on the set $\operatorname{Arc}(\Gamma)$ of arcs of $\Gamma$, then $\Gamma$ is said to be a $G$-symmetric graph, where an $\operatorname{arc}$ of $\Gamma$ is an ordered pair of adjacent vertices of $\Gamma$. In the following we will assume without mentioning explicitly that $\Gamma$ is nontrivial, that is, $\operatorname{Arc}(\Gamma) \neq \varnothing$. Then $\Gamma$ contains no isolated vertices since it is required to be $G$-vertex-transitive. Roughly speaking, in most cases $G$ acts imprimitively on the vertex set of a $G$-symmetric graph $\Gamma$, that is, $V(\Gamma)$ admits a nontrivial $G$-invariant partition $\mathcal{B}$; in this case $\Gamma$ is called an imprimitive $G$-symmetric graph. From permutation group theory [2, Corollary 1.5A], this happens precisely when the stabiliser $G_{\alpha}:=\left\{g \in G: \alpha^{g}=\alpha\right\}$ of $\alpha$ in $G$ is not a maximal subgroup of $G$, where $\alpha \in V(\Gamma)$. A standard approach to studying imprimitive $G$-symmetric graphs $\Gamma$ is to analyse the quotient graph $\Gamma_{\mathcal{B}}$ of $\Gamma$ with respect to $\mathcal{B}$, which is defined to be the graph with

* Supported by a Discovery Project Grant (DP0558677) from the Australian Research Council and a Melbourne Early Career Researcher Grant from The University of Melbourne. 
vertex set $\mathcal{B}$ in which $B, C \in \mathcal{B}$ are adjacent if and only if there exist $\alpha \in B$ and $\beta \in C$ such that $\{\alpha, \beta\}$ is an edge of $\Gamma$. In the following we assume that $\Gamma_{\mathcal{B}}$ contains at least one edge, so that each block of $\mathcal{B}$ is an independent set of $\Gamma$ (see [1, Proposition 22.1] or [8]). Also, without loss of generality we assume that $\Gamma_{\mathcal{B}}$ is connected for otherwise $\Gamma$ must be disconnected and we may deal with its connected components individually. (However, the connectedness of $\Gamma$ is not required in this paper. Note that $\Gamma_{\mathcal{B}}$ can be connected when $\Gamma$ is disconnected. For example, if $\Gamma$ is a matching with at least two edges, then it is disconnected but the quotient graph with respect to the natural bipartition is connected.) This quotient graph $\Gamma_{\mathcal{B}}$ conveys a lot of information about the graph $\Gamma$ and inherits some properties of $\Gamma$. For example, $\Gamma_{\mathcal{B}}$ is $G$-symmetric under the induced action (possibly unfaithful) of $G$ on $\mathcal{B}$ [8, Lemma 1.1(a)]. Nevertheless, $\Gamma_{\mathcal{B}}$ does not determine $\Gamma$ completely since it does not tell us how adjacent blocks of $\mathcal{B}$ are joined by edges of $\Gamma$. To compensate for this shortage, we need [3] the "inter-block" subgraph induced by two adjacent blocks of $\mathcal{B}$. Let $\Gamma(\alpha):=\{\beta \in V(\Gamma):\{\alpha, \beta\} \in E(\Gamma)\}$, the neighbourhood of $\alpha$ in $\Gamma$. For each $B \in \mathcal{B}$, let

$$
\Gamma(B):=\bigcup_{\alpha \in B} \Gamma(\alpha)
$$

For adjacent blocks $B, C$ of $\mathcal{B}$, define $\Gamma[B, C]$ to be the subgraph of $\Gamma$ induced by $(\Gamma(C) \cap$ $B) \cup(\Gamma(B) \cap C)$. Then $\Gamma[B, C]$ is a bipartite graph with bipartition $\{\Gamma(C) \cap B, \Gamma(B) \cap C\}$ as $B$ and $C$ are both independent sets of $\Gamma$. Since $\Gamma_{\mathcal{B}}$ is $G$-symmetric, up to isomorphism, $\Gamma[B, C]$ is independent of the choice of adjacent blocks $B, C$ of $\mathcal{B}$. Let

$$
\Gamma_{\mathcal{B}}(B):=\left\{C \in \mathcal{B}:\{B, C\} \in E\left(\Gamma_{\mathcal{B}}\right)\right\}
$$

be the neighbourhood of $B$ in $\Gamma_{\mathcal{B}}$. To depict genuinely the structure of $\Gamma$ we also need a "cross-sectional" geometry [3], namely the incidence structure

$$
\mathcal{D}(B):=\left(B, \Gamma_{\mathcal{B}}(B), \mathrm{I}\right)
$$

in which $\alpha \mathrm{I} C$ for $\alpha \in B$ and $C \in \Gamma_{\mathcal{B}}(B)$ if and only if $\alpha \in \Gamma(C)$. Clearly, the set of points of $\mathcal{D}(B)$ incident with a block $C \in \Gamma_{\mathcal{B}}(B)$ is $\Gamma(C) \cap B$. We denote by $\Gamma_{\mathcal{B}}(\alpha)$ the set of blocks of $\mathcal{D}(B)$ incident with a point $\alpha \in B$, that is,

$$
\Gamma_{\mathcal{B}}(\alpha):=\left\{C \in \Gamma_{\mathcal{B}}(B): \alpha \in \Gamma(C)\right\}
$$

Denote

$$
v:=|B|, \quad r:=\left|\Gamma_{\mathcal{B}}(\alpha)\right|, \quad b:=\left|\Gamma_{\mathcal{B}}(B)\right|, \quad k:=|\Gamma(C) \cap B|, \quad s:=|\Gamma(\alpha) \cap C| .
$$

Since $\Gamma$ and $\Gamma_{\mathcal{B}}$ are $G$-symmetric, these parameters are all independent of the choice of adjacent blocks $B, C$ of $\mathcal{B}$ and the flag $(\alpha, C)$ of $\mathcal{D}(B)$. One can check that $\mathcal{D}(B)$ is a 1-(v, $k, r)$ design with $b$ blocks and, up to isomorphism, is independent of the choice of $B$. Also, the setwise stabiliser $G_{B}:=\left\{g \in G: B^{g}=B\right\}$ of $B$ in $G$ induces a group of automorphisms of $\mathcal{D}(B)$, and $G_{B}$ is transitive on the points, the blocks and the flags of 
$\mathcal{D}(B)$ [3]. Thus, the number of times a block $C$ of $\mathcal{D}(B)$ is repeated is independent of the choice of $B$ and $C$. We denote this number by $m$ and call it the multiplicity of $\mathcal{D}(B)$. In the following we will view $\mathcal{D}(B)$ as the 1- $(v, k, r)$ design with point set $B$ and blocks the subsets $\Gamma(C) \cap B$ of $B$, for $C \in \Gamma_{\mathcal{B}}(B)$, each repeated $m$ times. We will reserve the letters $v, r, b, k, s$ for the above-defined parameters with respect to $\mathcal{B}$. Then the valency of $\Gamma$ is equal to $r s$, and the valencies of $\Gamma_{\mathcal{B}}$ and $\Gamma[B, C]$ are $b$ and $s$, respectively. If $k=v, s=1$, then $\Gamma[B, C]$ is a perfect matching between $B$ and $C$, and in this case $\Gamma$ is a cover of $\Gamma_{\mathcal{B}}$. In general, if $k=v$, then following [6], $\Gamma$ is called a multicover of $\Gamma_{\mathcal{B}}$.

Thus, with any imprimitive $G$-symmetric graph $\Gamma$ and nontrivial $G$-invariant partition $\mathcal{B}$ of $V(\Gamma)$ we have associated three configurations, namely the quotient graph $\Gamma_{\mathcal{B}}$, the bipartite graph $\Gamma[B, C]$, and the 1-design $\mathcal{D}(B)$. Gardiner and Praeger [3] suggested that we may analyse the triple $\left(\Gamma_{\mathcal{B}}, \Gamma[B, C], \mathcal{D}(B)\right)$ in order to study $\Gamma$. This approach is a geometric one in the sense that it involves the "cross-sectional" geometry $\mathcal{D}(B)$. It has been proved to be very useful in studying imprimitive symmetric graphs, see [3-5, 7, 12-15]. Clearly, $G_{B}$ induces natural actions on $B$ and $\Gamma_{\mathcal{B}}(B)$. These "local actions" may have significant influence on the structure of $\Gamma$, and the analysis of them is fundamental to make effective use of the approach. For example, it was proved in [12] that, if the actions of $G_{B}$ on $B$ and $\Gamma_{\mathcal{B}}(B)$ are permutationally equivalent, then $\Gamma$ can be reconstructed from $\Gamma_{\mathcal{B}}$ and the action of $G$ on $\mathcal{B}$ via a simple construction, called the 3 -arc graph construction, which was first introduced in [7] in the case where $k=v-1 \geq 2$ and $\mathcal{D}(B)$ contains no repeated blocks. (For a group $G$ acting on two sets $\Omega$ and $\Delta$, the actions of $G$ on $\Omega$ and $\Delta$ are said to be permutationally equivalent if there exists a bijection $\psi: \Omega \rightarrow \Delta$ such that $\psi\left(\alpha^{g}\right)=(\psi(\alpha))^{g}$ for all $\alpha \in \Omega$ and $g \in G$.)

The purpose of this paper is to study actions induced by the kernels $G_{(B)}, G_{[B]}$ of the actions of $G_{B}$ on $B, \Gamma_{\mathcal{B}}(B)$, where by definition

$$
\begin{aligned}
& G_{(B)}:=\left\{g \in G_{B}: \alpha^{g}=\alpha \text { for each } \alpha \in B\right\} \\
& G_{[B]}:=\left\{g \in G_{B}: C^{g}=C \text { for each } C \in \Gamma_{\mathcal{B}}(B)\right\} .
\end{aligned}
$$

In particular, we will investigate the action of $G_{[B]}$ on $B$ and the actions of $G_{(B)}$ on $\Gamma_{\mathcal{B}}(B)$, $\Gamma(\alpha)$ and $\Gamma_{\mathcal{B}}(\alpha)$ (where $\alpha \in B$ ), and the influence of these "local actions" on the structure of $\Gamma$. For our purpose it seems natural to distinguish whether one of $G_{(B)}, G_{[B]}$ is a subgroup of the other. With respect to this we have the following (not necessarily exclusive) possibilities: (i) $G_{[B]} \leq G_{(B)}$; (ii) $G_{[B]} \leq G_{(B)}$; (iii) $G_{(B)} \leq G_{[B]}$; (iv) $G_{(B)} \not G_{[B]}$; (v) $G_{[B]} \not \leq G_{(B)}$ and $G_{(B)} \not G_{[B]}$. Setting $M=G_{(B)} G_{[B]}, M$ is a normal subgroup of $G_{B}$ and we have Figure 1 in the lattice of subgroups of $G_{B}$.

We will put our discussion in a general setting and consider the following subgroups of $G_{B}$. Let $d$ be the diameter of $\Gamma_{\mathcal{B}}$, that is, the longest distance between two vertices of $\Gamma_{\mathcal{B}}$. For each $i$ with $0 \leq i \leq d$, let $\Gamma_{\mathcal{B}}(i, B)$ denote the set of blocks of $\mathcal{B}$ with distance in $\Gamma_{\mathcal{B}}$ no more than $i$ from $B$. Then $G_{B}$ leaves $\Gamma_{\mathcal{B}}(i, B)$ invariant, that is, $C \in \Gamma_{\mathcal{B}}(i, B)$ implies $C^{g} \in \Gamma_{\mathcal{B}}(i, B)$ for any $g \in G_{B}$, and hence $G_{B}$ induces a natural action on $\Gamma_{\mathcal{B}}(i, B)$. The kernel of this action is

$$
G_{[i, B]}:=\left\{g \in G_{B}: C^{g}=C \text { for each } C \in \Gamma_{\mathcal{B}}(i, B)\right\} .
$$




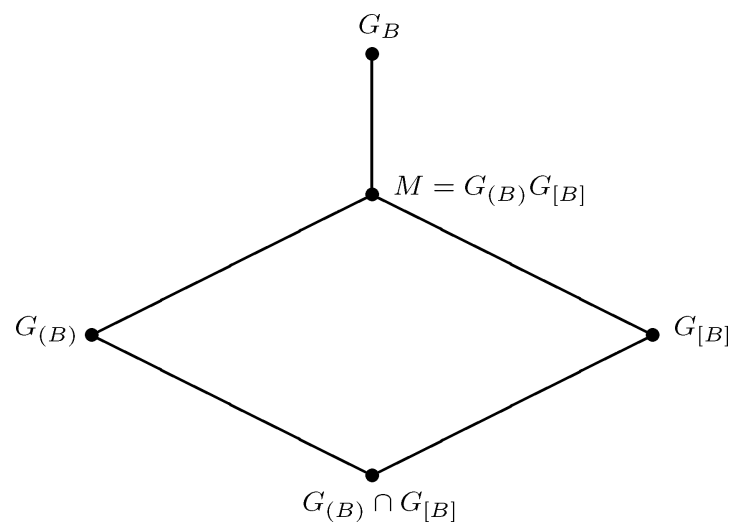

Figure 1. $G_{(B)}$ and $G_{[B]}$.

In particular, $G_{[0, B]}=G_{B}, G_{[1, B]}=G_{[B]}$, and $G_{[d, B]}$ coincides with the kernel of the induced action of $G$ on $\mathcal{B}$. Figure 2 illustrates the relationships among these groups $G_{[i, B]}$, $0 \leq i \leq d$.

The results obtained in this paper are generic in nature. In Section 2, we will show (Theorem 2.5) that each $G_{[i, B]}$ induces a $G$-invariant partition $\mathcal{B}_{i}$ of $V(\Gamma)$ such that the sequence

$$
\mathcal{B}=\mathcal{B}_{0}, \mathcal{B}_{1}, \mathcal{B}_{2}, \ldots, \mathcal{B}_{d}
$$

is a tower possessing some nice "level structure" properties, where as in [8] a sequence of $G$-invariant partitions is called a tower if each partition is a refinement of the previous partition. We will show (Theorem 2.7) further that, if $G_{[i, B]} \leq G_{(B)}$ for some $i \geq 1$ then $G$ is faithful on $\mathcal{B}$; whilst if $G_{[i, B]} \leq G_{(B)}$ for some $i \geq 1$ then either $\mathcal{B}_{i}$ is a genuine refinement of $\mathcal{B}$, or $\Gamma$ is a multicover of $\Gamma_{\mathcal{B}}$. (For two partitions $\mathcal{P}_{1}, \mathcal{P}_{2}$ of a set $\Omega$, we say that $\mathcal{P}_{1}$ is a refinement of $\mathcal{P}_{2}$ if each block of $\mathcal{P}_{2}$ is a union of some blocks of $\mathcal{P}_{1}$; and $\mathcal{P}_{1}$ is a genuine refinement of $\mathcal{P}_{2}$ if in addition $\mathcal{P}_{1} \neq\{\{\alpha\}: \alpha \in \Omega\}$ and $\mathcal{P}_{1} \neq \mathcal{P}_{2}$.) In Section 3 we will study an extreme case where any two blocks of $\mathcal{D}(B)$ are either repeated or disjoint, that is, for any $C, D \in \Gamma_{\mathcal{B}}(B)$, either $\Gamma(C) \cap B=\Gamma(D) \cap B$, or $\Gamma(C) \cap \Gamma(D) \cap B=\emptyset$. Based on these results, we then study in Section 4 the case where $\Gamma$ is $G$-locally quasiprimitive. (A $G$-symmetric graph $\Gamma$ is said to be $G$-locally quasiprimitive if $G_{\alpha}$ is quasiprimitive on $\Gamma(\alpha)$, that is, every non-identity normal subgroup of $G_{\alpha}$ is transitive on $\Gamma(\alpha)$.) In this case we will show (Theorem 4.2) amongst other things that, if $\mathcal{B}$ is a minimal $G$-invariant partition, then either $G_{[B]}=G_{(B)}$, or $k=1$ and $G_{[B]}<G_{(B)}$, or $\Gamma$ is a multicover of $\Gamma_{\mathcal{B}}$. For $\alpha \in V(\Gamma)$, we use $G_{[\alpha]}$ to denote the subgroup of $G_{\alpha}$ fixing setwise each block of $\Gamma_{\mathcal{B}}(\alpha)$, that is, $G_{[\alpha]}:=\left\{g \in G_{\alpha}: C^{g}=C\right.$ for each $\left.C \in \Gamma_{\mathcal{B}}(\alpha)\right\}$. Then $G_{[\alpha]}$ induces a natural action on $\Gamma(\alpha) \cap C$. In Section 4 we will also study $G$-locally quasiprimitive graphs $\Gamma$ such that $G_{[\alpha]}$ is transitive on $\Gamma(\alpha) \cap C$, and prove that in this case either $\Gamma$ is a bipartite graph or $\Gamma[B, C]$ is a matching. 


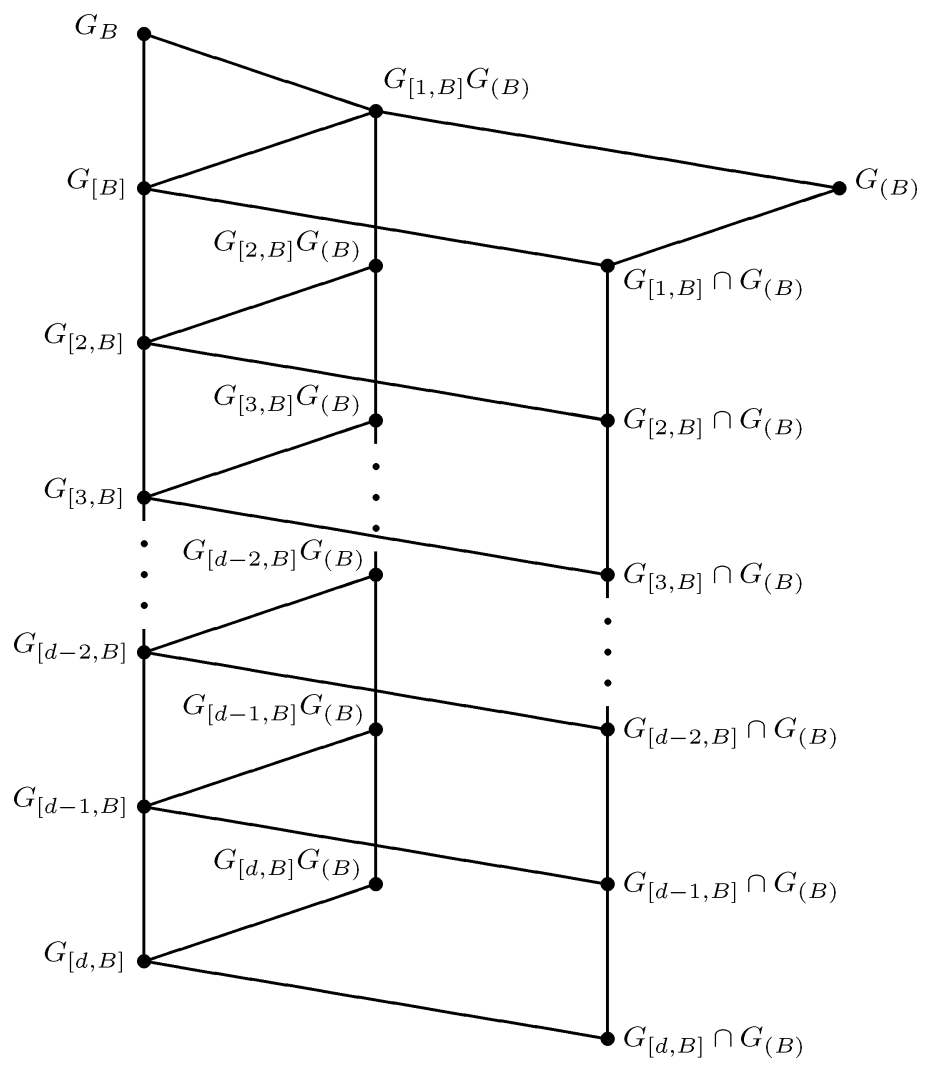

Figure 2. Relationships among $G_{[i, B]}$ 's.

\section{Tower induced by $G_{[i, B]}$}

We will follow standard terminology and notation for permutation groups, see e.g. [2]. For a $G$-invariant partition $\mathcal{B}$ of a finite set $\Omega$, each block $B$ of $\mathcal{B}$ is a block of imprimitivity for $G$ in $\Omega$ in the sense that, for each $g \in G$, either $B^{g}=B$ or $B^{g} \cap B=\emptyset$. Conversely, for a transitive group $G$ acting on $\Omega$, any block $B$ of imprimitivity for $G$ in $\Omega$ induces a $G$-invariant partition of $\Omega$, namely $\left\{B^{g}: g \in G\right\}$. As usual write $N \unlhd G$ if $N$ is a normal subgroup of $G$, and $N \triangleleft G$ if $N \unlhd G$ and $N \neq G$.

Lemma 2.1 (see e.g. [10, Lemma 10.1]) Let a group $G$ act on a finite set $\Omega$, and let $N \unlhd G$. Then the set of $N$-orbits on $\Omega$ is a $G$-invariant partition of $\Omega$.

We will denote this partition by $\mathcal{B}_{N}$ and, following [11], call it the $G$-normal partition of $\Omega$ induced by $N$. Clearly, for $G$ transitive on $\Omega$, the trivial partitions $\{\Omega\}$ and $\{\{\alpha\}: \alpha \in \Omega\}$ of $\Omega$ are $G$-normal partitions. If these are the only $G$-normal partitions of $\Omega$, then $G$ is said 
to be quasiprimitive on $\Omega$. Thus, $G$ is quasiprimitive on $\Omega$ if and only if every non-indentity normal subgroup of $G$ is transitive on $\Omega$.

Applying Lemma 2.1 to imprimitive symmetric graphs, we get the following result.

Lemma 2.2 Suppose $\Gamma$ is a $G$-symmetric graph admitting a nontrivial $G$-invariant partition $\mathcal{B}$, and let $B \in \mathcal{B}$. Then each normal subgroup $N$ of $G_{B}$ induces a $G$-invariant partition $\mathcal{B}_{N}^{*}$ of $V(\Gamma)$. Moreover, $\mathcal{B}_{N}^{*}$ is a refinement of $\mathcal{B}$ and the following (a)-(c) hold.

(a) $\mathcal{B}_{N}^{*}$ is the trivial partition $\{\{\alpha\}: \alpha \in V(\Gamma)\}$ if and only if $N \leq G_{(B)}$.

(b) $\mathcal{B}_{N}^{*}$ coincides with $\mathcal{B}$ if and only if $N$ is transitive on $B$.

(c) If $N \unlhd G$, then $\mathcal{B}_{N}^{*}$ coincides with the $G$-normal partition $\mathcal{B}_{N}$ of $V(\Gamma)$ induced by $N$.

Proof: Since $N \unlhd G_{B}$ and $G_{B}$ is transitive on $B$, Lemma 2.1 implies that $B^{*}:=\alpha^{N}$ is a block of imprimitivity for $G_{B}$ in $B$, where $\alpha \in B$. Since $\mathcal{B}$ is a $G$-invariant partition of $V(\Gamma)$, this implies that $B^{*}$ is a block of imprimitivity for $G$ in $V(\Gamma)$. Hence $B^{*}$ induces a $G$-invariant partition of $V(\Gamma)$, namely,

$$
\mathcal{B}_{N}^{*}:=\left\{\left(B^{*}\right)^{g}: g \in G\right\}
$$

The validity of (a)-(c) follows from the definition of $\mathcal{B}_{N}^{*}$ immediately.

\section{Remark 2.3}

(a) For distinct blocks $B, C \in \mathcal{B}$, there exists $g \in G$ such that $B^{g}=C$. So $\left(G_{B}\right)^{g}:=$ $g^{-1} G_{B} g=G_{C}$, and hence $N \unlhd G_{B}$ if and only if $N^{g}:=g^{-1} N g \unlhd G_{C}$. It is easy to see that $\mathcal{B}_{N^{g}}^{*}=\mathcal{B}_{N}^{*}$. So, in studying the $G$-invariant partition $\mathcal{B}_{N}^{*}$, we can start with any chosen block $B \in \mathcal{B}$.

(b) The results in Lemma 2.2 are valid for any transitive permutation group $G$ on a finite set $\Omega$, any nontrivial $G$-invariant partition $\mathcal{B}$ of $\Omega$ and any normal subgroup $N$ of $G_{B}$, where $B \in \mathcal{B}$. For the purpose of this paper, in Lemma 2.2 we stated these results in the case where $\Omega=V(\Gamma)$ and $G$ is a vertex- and arc-transitive group of automorphisms of $\Gamma$.

For adjacent blocks $B, C$ of $\mathcal{B}$, let $G_{B, C}:=\left(G_{B}\right)_{C}=\left\{g \in G: B^{g}=B, C^{g}=C\right\}$. Then $G_{B, C}$ is transitive on the set of edges of $\Gamma[B, C]$ ( [8, Lemma 1.4(b)]). From this it follows that

$$
\Gamma(C) \cap B \text { and } \Gamma(B) \cap C \text { are two }\left(G_{B, C}\right) \text {-orbits on } V(\Gamma) \text {. }
$$

This will be used in the proof of Theorem 2.5 below. Also, we will need the following observations, which can be easily verified.

Lemma 2.4 Suppose $\Gamma$ is a $G$-symmetric graph admitting a nontrivial $G$-invariant partition $\mathcal{B}$. Let $B \in \mathcal{B}$ and $\alpha \in B$, and let $d$ be the diameter of $\Gamma_{\mathcal{B}}$. Then the following (a)-(e) hold.

(a) $G_{(B)} \triangleleft G_{B}$. 
(b) $G_{(B)} \unlhd G_{\alpha}$.

(c) $G_{[\alpha]} \unlhd G_{\alpha}$.

(d) $G_{[i, B]} \unlhd G_{B}$ for each $i$ with $0 \leq i \leq d$; in particular, $G_{[B]} \unlhd G_{B}$.

(e) $G_{[i, B]} \unlhd G_{[i-1, B]}$ for each $i$ with $1 \leq i \leq d$.

Let $d$ be the diameter of $\Gamma_{\mathcal{B}}$. From Lemma 2.2 and Lemma 2.4(d) it follows that, for each $i$ with $0 \leq i \leq d, G_{[i, B]}$ induces a $G$-invariant partition

$$
\mathcal{B}_{i}:=\left\{B_{i}^{g}: g \in G\right\}
$$

of $V(\Gamma)$ which is a refinement of $\mathcal{B}$, where $B_{i}:=\alpha^{G_{[i, B]}}$ (for some $\alpha \in B$ ) is a typical block of $\mathcal{B}_{i}$. Let $v_{i}, r_{i}, b_{i}, k_{i}, s_{i}$ denote the parameters with respect to $\mathcal{B}_{i}$, as defined in (1). Since $\mathcal{B}_{0}$ is precisely the original partition $\mathcal{B}$, we have $\left(v_{0}, r_{0}, b_{0}, k_{0}, s_{0}\right)=(v, r, b, k, s)$. The following theorem gives some "level structure" properties concerning these partitions. Recall that a tower is a sequence of $G$-invariant partitions of $V(\Gamma)$ such that each partition in the sequence is a refinement of the previous partition.

Theorem 2.5 Suppose $\Gamma$ is a G-symmetric graph admitting a nontrivial $G$-invariant partition $\mathcal{B}$. Let $B \in \mathcal{B}$ and let $d$ be the diameter of $\Gamma_{\mathcal{B}}$. Then for each $i$ with $0 \leq i \leq d$, $G_{[i, B]}$ induces a $G$-invariant partition $\mathcal{B}_{i}$, defined in (4), such that $\mathcal{B}=\mathcal{B}_{0}, \mathcal{B}_{1}, \ldots, \mathcal{B}_{d}$ is a tower. Moreover, the following (a)-(d) hold.

(a) $v_{i}$ is a common divisor of $v_{i-1}$ and $k_{i-1}, s_{i}$ is a divisor of $s_{i-1}$, and $r_{i-1}$ is a divisor of $r_{i}$ (with $\left.s_{i-1} / s_{i}=r_{i} / r_{i-1}\right)$.

(b) Each block of the 1-design $\mathcal{D}\left(B_{i-1}\right)$ (for $B_{i-1} \in \mathcal{B}_{i-1}$ ) is a disjoint union of some blocks of $\mathcal{B}_{i}$. More precisely, for adjacent blocks $B_{i-1}, C_{i-1}$ of $\Gamma_{\mathcal{B}_{i-1}}, G_{[i, B]}$ leaves $\Gamma\left(C_{i-1}\right) \cap B_{i-1}$ invariant and the $\left(G_{[i, B]}\right)$-orbits on $\Gamma\left(C_{i-1}\right) \cap B_{i-1}$ form a $\left(G_{B_{i-1}, C_{i-1}}\right)$ invariant partition of $\Gamma\left(C_{i-1}\right) \cap B_{i-1}$.

(c) $\Gamma_{\mathcal{B}_{i-1}}(\alpha)=\Gamma_{\mathcal{B}_{i-1}}(\beta)$ for any vertices $\alpha, \beta$ in the same block of $\mathcal{B}_{i}$.

(d) For each integer $j$ with $0 \leq j<i$, the set $\mathcal{B}_{i}$ admits a $G$-invariant partition $\mathbf{B}_{i j}$ such that $\Gamma_{\mathcal{B}_{j}} \cong\left(\Gamma_{\mathcal{B}_{i}}\right)_{\mathbf{B}_{i j}}$ and that the parameters $\mathrm{v}_{i j}, \mathrm{r}_{i j}, \mathrm{~b}_{i j}, \mathrm{k}_{i j}, \mathrm{~s}_{i j}$ with respect to $\mathbf{B}_{i j}$ satisfy $\mathrm{v}_{i j}=v_{j} / v_{i}, \mathrm{k}_{i j}=k_{j} / v_{i}, \mathrm{~b}_{i j}=b_{j}, \mathrm{r}_{i j}=r_{j}, \mathrm{~s}_{i j}=b_{i} / r_{j}$.

Proof: For each $i$, let $\alpha \in B$ and $B_{i}:=\alpha^{G_{[i, B]}}$, and let $\mathcal{B}_{i}$ be as defined in (4). Then, since $G_{[i, B]} \unlhd G_{B}$ by Lemma 2.4(d), Lemma 2.2 implies that $\mathcal{B}_{i}$ is a $G$-invariant partition of $V(\Gamma)$ and is a refinement of $\mathcal{B}$. For $1 \leq i \leq d$, since $G_{[i, B]} \unlhd G_{[i-1, B]}$ (Lemma 2.4(e)), it follows that $\mathcal{B}_{i}$ is a refinement of $\mathcal{B}_{i-1}$. Consequently, $v_{i}$ is a divisor of $v_{i-1}$.

Now suppose $C_{i-1}$ is a block of $\mathcal{B}_{i-1}$ adjacent to $B_{i-1}$ in $\Gamma_{\mathcal{B}_{i-1}}$, and let $C$ be the block of $\mathcal{B}$ containing $C_{i-1}$. Then there exist $\beta \in \Gamma\left(C_{i-1}\right) \cap B_{i-1}$ and $\gamma \in \Gamma\left(B_{i-1}\right) \cap C_{i-1}$ such that $\beta, \gamma$ are adjacent in $\Gamma$. By the definition of $\mathcal{B}_{i-1}$, we have $B_{i-1}=\beta^{G_{i-1, B]}}$ and $C_{i-1}=$ $\gamma^{G_{[i-1, C]}}$, and by (3) we have $\Gamma\left(C_{i-1}\right) \cap B_{i-1}=\beta^{G_{B_{i-1},}, C_{i-1}}$ and $\Gamma\left(B_{i-1}\right) \cap C_{i-1}=\gamma^{G_{B_{i-1},}, C_{i-1}}$. Note that $B, C$ are adjacent blocks of $\mathcal{B}$. So we have $\Gamma_{\mathcal{B}}(i-1, C) \subseteq \Gamma_{\mathcal{B}}(i, B)$ and hence $G_{[i, B]} \leq G_{[i-1, C]}$. This implies that $G_{[i, B]}$ fixes $C_{i-1}$ setwise. Since $G_{[i, B]} \unlhd G_{[i-1, B]}, G_{[i, B]}$ also fixes $B_{i-1}$ setwise. Thus, we have $G_{[i, B]} \leq G_{B_{i-1}, C_{i-1}}$. This implies $G_{[i, B]} \unlhd G_{B_{i-1}, C_{i-1}}$ since $G_{B_{i-1}, C_{i-1}} \leq G_{B}$ and $G_{[i, B]} \unlhd G_{B}$ (Lemma 2.4(d)). So $G_{[i, B]}$ leaves $\Gamma\left(C_{i-1}\right) \cap B_{i-1}$ 
invariant and, by Lemma 2.1 , the $\left(G_{[i, B]}\right)$-orbits on $\Gamma\left(C_{i-1}\right) \cap B_{i-1}$ constitute a $\left(G_{B_{i-1}, C_{i-1}}\right)$ invariant partition of $\Gamma\left(C_{i-1}\right) \cap B_{i-1}$. Thus, each block $\Gamma\left(C_{i-1}\right) \cap B_{i-1}$ of the 1-design $\mathcal{D}\left(B_{i-1}\right)$ is a disjoint union of some blocks of $\mathcal{B}_{i}$. This implies in particular that $v_{i}$ is a divisor of $k_{i-1}$, and so $v_{i}$ is a common divisor of $v_{i-1}$ and $k_{i-1}$. One can see that each block $C_{i-1}$ of $\Gamma_{\mathcal{B}_{i-1}}(\beta)$ contains the same number of blocks of $\Gamma_{\mathcal{B}_{i}}(\beta)$. Hence $r_{i-1}$ is a divisor of $r_{i}$. Since $r_{i-1} s_{i-1}=r_{i} s_{i}$ (the valency of $\Gamma$ ), this implies that $s_{i}$ is a divisor of $s_{i-1}$.

If $\delta, \varepsilon$ are in the same block of $\mathcal{B}_{i}$, without loss of generality we may suppose that $\delta, \varepsilon \in B_{i}$. Then since $B_{i}$ is a $\left(G_{[i, B]}\right)$-orbit there exists $x \in G_{[i, B]}$ such that $\delta^{x}=\varepsilon$, and hence $\left(\Gamma_{\mathcal{B}_{i-1}}(\delta)\right)^{x}=\Gamma_{\mathcal{B}_{i-1}}(\varepsilon)$. On the other hand, the elements of $G_{[i, B]}$ fix setwise each block $C_{i-1}$ in $\Gamma_{\mathcal{B}_{i-1}}\left(B_{i-1}\right)$ since $G_{[i, B]} \unlhd G_{B_{i-1}, C_{i-1}}$, as shown above. In particular, $x$ fixes setwise each block in $\Gamma_{\mathcal{B}_{i-1}}(\delta)$ since $\Gamma_{\mathcal{B}_{i-1}}(\delta) \subseteq \Gamma_{\mathcal{B}_{i-1}}\left(B_{i-1}\right)$. Thus, we have $\Gamma_{\mathcal{B}_{i-1}}(\delta)=$ $\left(\Gamma_{\mathcal{B}_{i-1}}(\delta)\right)^{x}=\Gamma_{\mathcal{B}_{i-1}}(\varepsilon)$.

Let $j$ be an integer with $0 \leq j<i$. Since for each $\ell$ with $j+1 \leq \ell \leq i$ the partition $\mathcal{B}_{\ell}$ is a refinement of the partition $\mathcal{B}_{\ell-1}$, as shown above, we know that $\mathcal{B}_{i}$ is a refinement of $\mathcal{B}_{j}$ and hence each block $C_{j}$ of $\mathcal{B}_{j}$ is a union of some blocks of $\mathcal{B}_{i}$. Denote by $\mathbf{C}_{i j}=\left\{B_{i}^{z}\right.$ : $\left.B_{i}^{z} \subseteq C_{j}, z \in G\right\}$, the set of blocks of $\mathcal{B}_{i}$ contained in $C_{j}$. Then $\mathbf{B}_{i j}:=\left\{\mathbf{C}_{i j}: C_{j} \in \mathcal{B}_{j}\right\}$ is a partition of $\mathcal{B}_{i}$. We claim further that $\mathbf{B}_{i j}$ is a $G$-invariant partition of $\mathcal{B}_{i}$ under the induced action of $G$ on $\mathcal{B}_{i}$. In fact, if $\mathbf{C}_{i j}^{g} \cap \mathbf{C}_{i j} \neq \varnothing$ for some $g \in G$, say $\left(B_{i}^{x}\right)^{g}=B_{i}^{y}$ for some $B_{i}^{x}, B_{i}^{y} \in \mathbf{C}_{i j}$, then $B_{i}^{x}, B_{i}^{y} \subseteq C_{j}$ and hence $\left(B_{i}^{x}\right)^{g}=B_{i}^{y} \subseteq C_{j}$. Since $C_{j}$ is a block of imprimitivity for $G$ in $V(\Gamma)$, this implies that $g$ fixes $C_{j}$ setwise. Therefore, we have $\mathbf{C}_{i j}^{g}=\left\{\left(B_{i}^{z}\right)^{g}: B_{i}^{z} \subseteq C_{j}, z \in G\right\}=\mathbf{C}_{i j}$ and hence $\mathbf{B}_{i j}$ is $G$-invariant indeed. Clearly, the mapping $\psi: C_{j} \mapsto \mathbf{C}_{i j}$ is a bijection from $\mathcal{B}_{j}$ to $\mathbf{B}_{i j}$. By the definition of a quotient graph, one can see that $\psi$ is an isomorphism from $\Gamma_{\mathcal{B}_{j}}$ to $\left(\Gamma_{\mathcal{B}_{i}}\right)_{\mathbf{B}_{i j}}$, and hence $\Gamma_{\mathcal{B}_{j}} \cong\left(\Gamma_{\mathcal{B}_{i}}\right)_{\mathbf{B}_{i j}}$. Clearly, we have $\mathrm{v}_{i j}=v_{j} / v_{i}, \mathrm{k}_{i j}=k_{j} / v_{i}, \mathrm{~b}_{i j}=b_{j}$ and $\mathrm{r}_{i j} \mathrm{~s}_{i j}=\operatorname{val}\left(\Gamma_{\mathcal{B}_{i}}\right)=b_{i}$. From $\mathrm{v}_{i j} \mathrm{r}_{i j}=\mathrm{b}_{i j} \mathrm{k}_{i j}$, we get $\left(v_{j} / v_{i}\right) \mathrm{r}_{i j}=b_{j}\left(k_{j} / v_{i}\right)$, which in turn implies $\mathrm{r}_{i j}=r_{j}$ since $v_{j} r_{j}=b_{j} k_{j}$. Finally, we have $\mathrm{s}_{i j}=b_{i} / r_{i j}=b_{i} / r_{j}$ and the proof is complete.

Remark 2.6 If $G_{[i, B]} \unlhd G$ for $B \in \mathcal{B}$, then from Lemma 2.2(c), $\mathcal{B}_{i}$ is the $G$-normal partition of $V(\Gamma)$ induced by $G_{[i, B]}$. In this case $\Gamma$ is a multicover of $\Gamma_{\mathcal{B}_{i}}$ (see [8, Section 1] or [11, Theorem 4.1]). In particular, if $\Gamma_{\mathcal{B}}$ is a complete graph, then $d=1$ and $G_{[B]} \unlhd G$ (since $G_{[B]}$ is the kernel of the action of $G$ on $\mathcal{B}$ in this case), and hence $\Gamma$ is a multicover of $\Gamma_{\mathcal{B}_{1}}$.

Theorem 2.7 Suppose $\Gamma$ is a $G$-symmetric graph admitting a nontrivial $G$-invariant partition $\mathcal{B}$, where $G \leq \operatorname{Aut}(\Gamma)$. Let $B \in \mathcal{B}$ and let $d$ be the diameter of $\Gamma_{\mathcal{B}}$. Then one of the following (a)-(b) occurs for each $i$ with $1 \leq i \leq d$.

(a) $G_{[i, B]} \leq G_{(B)}$; in this case $G$ is faithful on $\mathcal{B}$.

(b) $G_{[i, B]} \not \leq G_{(B)}$; in this case either

(i) $G_{[i, B]}$ induces a $G$-invariant partition $\mathcal{B}_{i}$ of $V(\Gamma)$, defined in $(4)$, which is a genuine refinement of $\mathcal{B}$ and is such that $v_{i}$ is a common divisor of $v$ and $k, s_{i}$ is a divisor of $s$, and $r$ is a divisor of $r_{i}$; or

(ii) $\Gamma$ is a multicover of $\Gamma_{\mathcal{B}}$ and $G_{[i, B]}$ is transitive on $B$.

Proof: Suppose that $G_{[i, B]} \leq G_{(B)}$. Then, since $G$ is transitive on $\mathcal{B}$ and since $G_{\left[i, B^{g}\right]}=$ $\left(G_{[i, B]}\right)^{g}$ and $G_{\left(B^{g}\right)}=\left(G_{(B)}\right)^{g}$ for any $g \in G$, we have $G_{[i, C]} \leq G_{(C)}$ for all blocks $C \in \mathcal{B}$. 
Thus, if $g$ is in the kernel of the action of $G$ on $\mathcal{B}$, then $g \in G_{[i, C]}$ in particular and hence $g \in G_{(C)}$. In other words, $g$ fixes each vertex in $C$. Since this holds for all $C \in \mathcal{B}$, it follows that $g$ fixes each vertex of $\Gamma$. Thus, since $G \leq \operatorname{Aut}(\Gamma)$ is faithful on $V(\Gamma)$, we have $g=1$ and hence $G$ is faithful on $\mathcal{B}$ as well.

Now suppose $G_{[i, B]} \not \leq G_{(B)}$. Then, by Lemma 2.2(a), the partition $\mathcal{B}_{i}$ of $V(\Gamma)$ induced by $G_{[i, B]}$ is a nontrivial $G$-invariant partition of $V(\Gamma)$. So we know from Lemma 2.2(b) and Theorem 2.5 that, either $\mathcal{B}_{i}$ is a genuine refinement of $\mathcal{B}$, or $G_{[i, B]}$ is transitive on $B$. In the former case, it follows from Theorem 2.5(a) that $v_{i}$ is a common divisor of $v$ and $k, s_{i}$ is a divisor of $s$ and $r$ is a divisor of $r_{i}$, and hence (i) in (b) occurs. Since $G_{[i, B]}$ fixes setwise the block $B$ and each block $C \in \Gamma_{\mathcal{B}}(B)$, it also fixes setwise $\Gamma(C) \cap B$. So in the latter case where $G_{[i, B]}$ is transitive on $B$, we must have $\Gamma(C) \cap B=B$, that is, $\Gamma$ is a multicover of $\Gamma_{\mathcal{B}}$ and hence (ii) in (b) occurs.

Note that, if case (b)(i) in Theorem 2.7(b) occurs, then at least one of the $\mathbf{B}_{i j}$ given in Theorem 2.5(d), say $\mathbf{B}_{i 0}$, is a nontrivial partition of $\mathcal{B}_{i}$. If case (b)(ii) in Theorem 2.7(b) occurs, then from Lemma $2.2(\mathrm{~b})$, the partition $\mathcal{B}_{i}$ induced by $G_{[i, B]}$ coincides with $\mathcal{B}$. Applying Theorem 2.7 to $G_{[B]}$, we get the following consequence.

Corollary 2.8 Suppose $(\Gamma, G, \mathcal{B})$ is as in Theorem 2. Then one of the following (a)-(b) occurs.

(a) $G_{[B]} \leq G_{(B)}$; in this case $G$ is faithful on $\mathcal{B}$.

(b) $G_{[B]} \not \leq G_{(B)}$; in this case either

(i) $G_{[B]}$ induces a $G$-invariant partition of $V(\Gamma)$, namely $\mathcal{B}_{1}$ defined in (4) for $i=1$, which is a genuine refinement of $\mathcal{B}$ such that $v_{1}$ is a common divisor of $v$ and $k$, $s_{1}$ is a divisor of $s$, and $r$ is a divisor of $r_{1}$; or

(ii) $\Gamma$ is a multicover of $\Gamma_{\mathcal{B}}$ and $G_{[B]}$ is transitive on $B$.

If the vertices in $B$ are "distinguishable" in the sense that $\Gamma_{\mathcal{B}}(\alpha) \neq \Gamma_{\mathcal{B}}(\beta)$ for distinct $\alpha, \beta \in B$, then case (a) in Corollary 2.8 occurs. In particular, this happens for $G$-symmetric graphs with $k=v-1 \geq 1$, see [7, Theorems 4 and 5(d)]. A nontrivial $G$-invariant partition $\mathcal{B}$ of $V(\Gamma)$ is said to be minimal if there is no $G$-invariant partition of $V(\Gamma)$ which is a genuine refinement of $\mathcal{B}$. For such a partition $\mathcal{B}$, case (b)(i) in Corollary 2.8 does not appear. The following example shows that case (b)(ii) in Corollary 2.8 occurs if $G$ is not quasiprimitive on $V(\Gamma)$ and if $\mathcal{B}$ is a nontrivial $G$-normal partition of $V(\Gamma)$.

Example 2.9 Suppose $\Gamma$ is a $G$-symmetric graph such that $G$ is not quasiprimitive on $V(\Gamma)$, where $G \leq \operatorname{Aut}(\Gamma)$. Then there exists a nontrivial normal subgroup $N$ of $G$ which is intransitive on $V(\Gamma)$, so the $G$-normal partition $\mathcal{B}_{N}$ of $V(\Gamma)$ induced by $N$ (Lemma 2.1) is nontrivial. Let $\Gamma_{N}$ be the quotient graph of $\Gamma$ with respect to $\mathcal{B}_{N}$. Since $N$ is contained in the kernel of the action of $G$ on $\mathcal{B}_{N}, G$ is not faithful on $\mathcal{B}_{N}$. So from Corollary 2.8 we must have $G_{[B]} \not \leq G_{(B)}$ for $B \in \mathcal{B}_{N}$. Since $N \unlhd G_{[B]}$, we have $B=\alpha^{N} \subseteq \alpha^{G_{[B]}} \subseteq B$ for $\alpha \in B$, which implies $\alpha^{G_{[B]}}=B$. Hence $G_{[B]}$ is transitive on $B$, and consequently we come to the result (see e.g. [11 Theorem 4.1]) that $\Gamma$ is a multicover of $\Gamma_{N}$. Thus, case (b)(ii) in Corollary 2.8 occurs. 


\section{Analysing an extreme case}

In Corollary 2.8 we have shown that, if $G_{[B]} \not \leq G_{(B)}$, then either $\Gamma$ is a multicover of $\Gamma_{\mathcal{B}}$, or we get a genuine refinement of $\mathcal{B}$. Note that $G_{B}$ is transitive on $\Gamma_{\mathcal{B}}(B)$ and $G_{(B)} \triangleleft G_{B}$ by Lemma 2.4(a). So in the opposite case where $G_{(B)} \not \leq G_{[B]}$, Lemma 2.1 implies that the $G_{(B)}$-orbits on $\Gamma_{\mathcal{B}}(B)$ form a nontrivial $G_{B}$-invariant partition of $\Gamma_{\mathcal{B}}(B)$. Since $G_{(B)}$ fixes $B$ pointwise, any two blocks in the same $G_{(B)}$-orbit on $\Gamma_{\mathcal{B}}(B)$ induce repeated blocks of $\mathcal{D}(B)$. In some cases, blocks in distinct $G_{(B)}$-orbits on $\Gamma_{\mathcal{B}}(B)$ may induce disjoint blocks of $\mathcal{D}(B)$. For example, in Remark 3.2 below we will see that this happens in particular when $\Gamma$ is $G$-locally quasiprimitive and $G_{(B)} \not \leq G_{[B]}$. This motivated us to study the case where, for any $C, D \in \Gamma_{\mathcal{B}}(B)$, either $\Gamma(C) \cap B=\Gamma(D) \cap B$, or $\Gamma(C) \cap \Gamma(D) \cap B=\emptyset$. In this case, the multiplicity $m$ of $\mathcal{D}(B)$ is equal to $r$. This seemingly trivial case is by no means trivial because it contains the following two very difficult but important subcases:

(i) $k=1$;

(ii) $k=v$.

We have studied the first subcase in [14, Section 4], where we gave a construction of such graphs from certain kinds of $G$-point- and $G$-block-transitive 1-designs. In the second subcase, $\Gamma$ is a multicover of $\Gamma_{\mathcal{B}}$. Our study in this section shows that (see Remark 3(a) below), in some sense, the study of $G$-symmetric graphs with blocks $\Gamma(C) \cap B$ of $\mathcal{D}(B)$ (for $C \in \Gamma_{\mathcal{B}}(B)$ ) satisfying the condition above can be reduced to the study of these two subcases. The results obtained here will be used in the next section. Define $\left(G_{B}\right)_{\Gamma_{\mathcal{B}}(\alpha)}:=$ $\left\{g \in G_{B}:\left(\Gamma_{\mathcal{B}}(\alpha)\right)^{g}=\Gamma_{\mathcal{B}}(\alpha)\right\}$.

Lemma 3.1 Suppose $\Gamma$ is a $G$-symmetric graph admitting a nontrivial $G$-invariant partition $\mathcal{B}$. Let $B \in \mathcal{B}, \alpha \in B$, and let (a), (b), (c) be the following statements. Then (a) implies (b), and (b) in turn implies (c).

(a) $G_{(B)} \not \leq G_{[B]}$, and either $G_{\alpha}$ or $\left(G_{B}\right)_{\Gamma_{\mathcal{B}}(\alpha)}$ is quasiprimitive on $\Gamma_{\mathcal{B}}(\alpha)$;

(b) $G_{(B)}$ is transitive on $\Gamma_{\mathcal{B}}(\alpha)$;

(c) either $\Gamma(C) \cap B=\Gamma(D) \cap B$ or $\Gamma(C) \cap \Gamma(D) \cap B=\emptyset$, for $C, D \in \Gamma_{\mathcal{B}}(B)$.

Proof: (a) $\Rightarrow$ (b) Suppose $G_{(B)} \not \leq G_{[B]}$. Then there exist $x \in G_{(B)}$ and $C, D \in \Gamma_{\mathcal{B}}(B)$ with $C \neq D$ such that $C^{x}=D$. Let $\alpha \in \Gamma(C) \cap B$, so that $C \in \Gamma_{\mathcal{B}}(\alpha)$. Since $x$ fixes each vertex in $B$ and hence fixes $\alpha$ in particular, we have $(\Gamma(\alpha) \cap C)^{x}=\Gamma(\alpha) \cap D$. Since $\Gamma(\alpha) \cap C \neq \emptyset$, we have $\Gamma(\alpha) \cap D \neq \emptyset$ and hence $D \in \Gamma_{\mathcal{B}}(\alpha)$. Thus the action of $G_{(B)}$ on $\Gamma_{\mathcal{B}}(\alpha)$ is nontrivial. On the other hand, since $G_{(B)} \triangleleft G_{B}$ (Lemma 2.4(a)) and $G_{(B)} \leq\left(G_{B}\right)_{\Gamma_{\mathcal{B}}(\alpha)} \leq G_{B}$, we have $G_{(B)} \unlhd\left(G_{B}\right)_{\Gamma_{\mathcal{B}}(\alpha)}$. So if $\left(G_{B}\right)_{\Gamma_{\mathcal{B}}(\alpha)}$ is quasiprimitive on $\Gamma_{\mathcal{B}}(\alpha)$, then $G_{(B)}$ must be transitive on $\Gamma_{\mathcal{B}}(\alpha)$. Similarly, since $G_{(B)} \unlhd G_{\alpha}$ (Lemma 2.4(b)) and $G_{(B)}$ acts on $\Gamma_{\mathcal{B}}(\alpha)$ in a nontrivial way, the quasiprimitivity of $G_{\alpha}$ on $\Gamma_{\mathcal{B}}(\alpha)$ implies the transitivity of $G_{(B)}$ on $\Gamma_{\mathcal{B}}(\alpha)$.

(b) $\Rightarrow$ (c) The assumption in (b) implies that, for any $\beta \in B, G_{(B)}$ is transitive on $\Gamma_{\mathcal{B}}(\beta)$. In fact, since $G_{B}$ is transitive on $B$, there exists $g \in G_{B}$ such that $\beta^{g}=\alpha$. For any $C, D \in \Gamma_{\mathcal{B}}(\beta)$, we have $C^{g}, D^{g} \in \Gamma_{\mathcal{B}}(\alpha)$ and hence by (b) there exists $x \in G_{(B)}$ 
such that $\left(C^{g}\right)^{x}=D^{g}$, that is, $C^{g x g^{-1}}=D$. Since $G_{(B)} \triangleleft G_{B}$ by Lemma 2.4(a), we have $g x g^{-1} \in G_{(B)}$ and hence $G_{(B)}$ is transitive on $\Gamma_{\mathcal{B}}(\beta)$ indeed. Also, since $C^{g x g^{-1}}=D$, we have $(\Gamma(C) \cap B)^{g x g^{-1}}=\Gamma(D) \cap B$. However, $g x g^{-1} \in G_{(B)}$ fixes each vertex in $B$, so we have $(\Gamma(C) \cap B)^{\text {gxg }^{-1}}=\Gamma(C) \cap B$ and consequently $\Gamma(C) \cap B=\Gamma(D) \cap B$. In other words, if two blocks $\Gamma(C) \cap B, \Gamma(D) \cap B$ of $\mathcal{D}(B)$ have a common vertex $\beta$, then $\Gamma(C) \cap B=\Gamma(D) \cap B$. Hence (c) is true.

Remark 3.2 Clearly, the quasiprimitivity of $G_{\alpha}$ on $\Gamma(\alpha)$ implies the quasiprimitivity of $G_{\alpha}$ on $\Gamma_{\mathcal{B}}(\alpha)$. So, if $\Gamma$ is a $G$-locally quasiprimitive graph admitting a nontrivial $G$-invariant partition $\mathcal{B}$ such that $G_{(B)} \not \leq G_{[B]}$, then by Lemma 3.1, either $\Gamma(C) \cap B=\Gamma(D) \cap B$ or $\Gamma(C) \cap \Gamma(D) \cap B=\emptyset$, for any $C, D \in \Gamma_{\mathcal{B}}(B)$.

The main result in this section is the following theorem.

Theorem 3.3 Suppose $\Gamma$ is a $G$-symmetric graph admitting a nontrivial $G$-invariant partition $\mathcal{B}$. Suppose further that, for any $C, D \in \Gamma_{\mathcal{B}}(B)$, either $\Gamma(C) \cap B=\Gamma(D) \cap B$ or $\Gamma(C) \cap \Gamma(D) \cap B=\emptyset$. Then $V(\Gamma)$ admits a second $G$-invariant partition $\mathcal{B}^{*}:=\left\{\left(B^{*}\right)^{g}\right.$ : $g \in G\}$, where $B^{*}$ is a block of $\mathcal{D}(B)$. Moreover, the following (a)-(c) hold.

(a) $\mathcal{B}^{*}$ is a refinement of $\mathcal{B}$, and it is a genuine refinement of $\mathcal{B}$ if and only if $2 \leq k \leq v-1$.

(b) $\Gamma$ is a multicover of $\Gamma_{\mathcal{B}^{*}}, k$ is a divisor of $v$, and the parameters $v^{*}, r^{*}, b^{*}, k^{*}, s^{*}$ with respect to $\mathcal{B}^{*}$ satisfy $v^{*}=k^{*}=k, b^{*}=r^{*}=r, s^{*}=s$.

(c) There exists a $G$-invariant partition $\mathbf{B}$ of $\mathcal{B}^{*}$ such that $\left(\Gamma_{\mathcal{B}^{*}}\right)_{\mathbf{B}} \cong \Gamma_{\mathcal{B}}$ and the parameters $\mathrm{v}, \mathrm{r}, \mathrm{b}, \mathrm{k}, \mathrm{s}$ with respect to $\mathbf{B}$ satisfy $\mathrm{v}=v / v^{*}, \mathrm{k}=\mathrm{s}=1, \mathrm{~b}=b$ and $\mathrm{r}=r$.

Proof: Our assumption on $\mathcal{D}(B)$ implies that the set of subsets of $B$ of the form $\Gamma(C) \cap B$, for $C \in \Gamma_{\mathcal{B}}(B)$, is a partition of $B$, which we denote by $\mathcal{P}(B)$. Thus the blocks of $\mathcal{P}(B)$ have size $k$ and $k$ divides $v$. Let $B^{*}:=\Gamma(C) \cap B$ be a typical block of $\mathcal{P}(B)$, where $C \in \Gamma_{\mathcal{B}}(B)$. Since $G_{B}$ is transitive on $\Gamma_{\mathcal{B}}(B)$ and since $\left(B^{*}\right)^{g}=\Gamma\left(C^{g}\right) \cap B$ for $g \in G_{B}$, we have $\mathcal{P}(B)=\left\{\left(B^{*}\right)^{g}: g \in G_{B}\right\}$ and hence $\mathcal{P}(B)$ is a $G_{B}$-invariant partition of $B$. We claim further that $\mathcal{B}^{*}:=\left\{\left(B^{*}\right)^{g}: g \in G\right\}$ defines a $G$-invariant partition of $V(\Gamma)$. In fact, if $\left(B^{*}\right)^{g} \cap B^{*} \neq \emptyset$ for some $g \in G$, then $B^{g} \cap B \neq \emptyset$ since $B^{*} \subseteq B$ and $\left(B^{*}\right)^{g} \subseteq B^{g}$. But $B$ is a block of imprimitivity for $G$ in $V(\Gamma)$, so we have $B^{g}=B$ and hence $g \in G_{B}$. Thus $\left(B^{*}\right)^{g} \subseteq B$ and $\left(B^{*}\right)^{g}$ is a block of $\mathcal{P}(B)$ having nonempty intersection with $B^{*}$. Since $\mathcal{P}(B)$ is a $G_{B}$-invariant partition of $B$, as shown above, this implies $\left(B^{*}\right)^{g}=B^{*}$. Therefore, $B^{*}$ is a block of imprimitivity for $G$ in $V(\Gamma)$ and so $\mathcal{B}^{*}$ is a $G$-invariant partition of $V(\Gamma)$. It is easily checked that $\mathcal{B}^{*}=\bigcup_{B \in \mathcal{B}} \mathcal{P}(B)$. Clearly, $\mathcal{B}^{*}$ is a refinement of $\mathcal{B}$, and it is a genuine refinement of $\mathcal{B}$ if and only if $2 \leq k \leq v-1$. Since $\Gamma_{\mathcal{B}}$ is $G$-symmetric, there exists $h \in G$ which interchanges $B$ and $C$. So $\Gamma(B) \cap C=(\Gamma(C) \cap B)^{h}=\left(B^{*}\right)^{h} \in \mathcal{B}^{*}$, and hence each vertex in $B^{*}$ is adjacent to at least one vertex in $\left(B^{*}\right)^{h}$. Therefore, $\Gamma$ is a multicover of $\Gamma_{\mathcal{B}^{*}}$, and hence $v^{*}=k^{*}=k, b^{*}=r^{*}=r, s^{*}=s$. Finally, it is straightforward to show that $\mathbf{B}:=\{\mathcal{P}(B): B \in \mathcal{B}\}$ is a $G$-invariant partition of $\mathcal{B}^{*}$ and that $\left(\Gamma_{\mathcal{B}^{*}}\right)_{\mathbf{B}} \cong \Gamma_{\mathcal{B}}$. Also, it is clear that the parameters $v, r, b, k, s$ with respect to $\mathbf{B}$ are as specified in (c). 


\section{Remark 3.4}

(a) The partition $\mathcal{B}^{*}$ in Theorem 3.3 is equal to the trivial partition $\{\{\alpha\}: \alpha \in V(\Gamma)\}$ if and only if $k=1$, and is equal to $\mathcal{B}$ if and only if $k=v$. In the general case where $2 \leq k \leq v-1, \mathcal{B}^{*}$ is a genuine refinement of $\mathcal{B}$, and as $k^{*}=v^{*}$, the partition $\left(\mathcal{B}^{*}\right)^{*}$ resulting from applying Theorem 3.3 to $\mathcal{B}^{*}$, is equal to $\mathcal{B}^{*}$. Moreover, the quotient graph $\Gamma_{\mathcal{B}^{*}}$ admits a $G$-invariant partition, namely $\mathbf{B}$, for which $k=1$ and thus the construction given in [14, Section 4] applies to $\Gamma_{\mathcal{B}^{*}}$.

(b) Setting $i=1$ in Theorem 2.5(b), we know that the partition $\mathcal{B}_{1}$ (defined in (4) for $i=1$ ) is a refinement of $\mathcal{B}^{*}$. Moreover, $\mathcal{B}_{1}$ admits a $G$-invariant partition $\mathbf{B}_{1}:=\left\{\mathcal{P}\left(B^{*}\right): B^{*} \in\right.$ $\left.\mathcal{B}^{*}\right\}$, where $\mathcal{P}\left(B^{*}\right):=\left\{\alpha^{G_{[B]}} \subseteq B^{*}: \alpha \in B^{*}\right\}$, such that $\left(\Gamma_{\mathcal{B}_{1}}\right)_{\mathbf{B}_{1}} \cong \Gamma_{\mathcal{B}^{*}}$ and $\Gamma_{\mathcal{B}_{1}}$ is a multicover of $\Gamma_{\mathcal{B}^{*}}$, and that the parameters $\mathrm{v}_{1}, \mathrm{r}_{1}, \mathrm{~b}_{1}, \mathrm{k}_{1}, \mathrm{~s}_{1}$ with respect to $\mathbf{B}_{1}$ satisfy $\mathrm{v}_{1}=\mathrm{k}_{1}=k / v_{1}, \mathrm{r}_{1}=\mathrm{b}_{1}=r, \mathrm{~s}_{1}=b_{1} / r$.

\section{Locally quasiprimitive graphs}

We now apply the results obtained in the last two sections to $G$-locally quasiprimitive graphs. Such graphs were studied initially in $[8,9]$, and more recent results were obtained in [6]. The following theorem is a generalization of [3, Lemma 3.4], where $\Gamma$ is required to be $G$-locally primitive (that is, $G_{\alpha}$ is primitive on $\Gamma(\alpha)$ ).

Theorem 4.1 Suppose $\Gamma$ is a G-locally quasiprimitive graph admitting a nontrivial $G$ invariant partition $\mathcal{B}$. Then one of the following (a)-(c) holds.

(a) $G_{[B]}=G_{(B)}$

(b) $G_{(B)} \not \leq G_{[B]}$; in this case $G_{(B)}$ is transitive on $\Gamma(\alpha)$ for each $\alpha \in B$, and moreover either

(i) $k=1$ and $G_{[B]}<G_{(B)}$; or

(ii) $k \geq 2, k$ divides $v$, and $V(\Gamma)$ admits a second nontrivial $G$-invariant partition $\mathcal{B}^{*}$ such that $\mathcal{B}^{*}$ is a refinement of $\mathcal{B}, \Gamma$ is a multicover of $\Gamma_{\mathcal{B}^{*}}$ and the parameters $v^{*}, r^{*}, b^{*}, k^{*}, s^{*}$ with respect to $\mathcal{B}^{*}$ satisfy $v^{*}=k^{*}=k, b^{*}=r^{*}=r, s^{*}=s$.

(c) $G_{[B]} \not \leq G_{(B)}$; in this case $G_{[B]}$ induces a nontrivial $G$-invariant partition $\mathcal{B}_{1}$ of $V(\Gamma)$ (defined in (4) for $i=1$ ) such that $\mathcal{B}_{1}$ is a refinement of $\mathcal{B}, v_{1}$ is a common divisor of $v$ and $k, s_{1}$ is a divisor of $s$, and $r$ is a divisor of $r_{1}$.

Proof: Suppose $G_{(B)} \not \leq G_{[B]}$. Then there exist $x \in G_{(B)}$ and distinct blocks $C, D$ of $\Gamma_{\mathcal{B}}(B)$ such that $C^{x}=D$. Let $\beta \in \Gamma(C) \cap B$, so that $\Gamma(\beta) \cap C \neq \emptyset$. Since $x$ fixes each vertex in $B$, it fixes $\beta$ in particular and hence maps a vertex in $\Gamma(\beta) \cap C$ to a vertex in $\Gamma(\beta) \cap D$. Since $G_{(B)} \unlhd G_{\beta}$ (Lemma 2.4(b)), this implies that $G_{(B)}^{\Gamma(\beta)}$ is a nontrivial normal subgroup of $G_{\beta}^{\Gamma(\beta)}$. Therefore, by the $G$-local quasiprimitivity of $\Gamma$, we conclude that $G_{(B)}$ is transitive on $\Gamma(\beta)$. Now for any $\alpha \in B$ there exists $g \in G_{B}$ such that $\alpha^{g}=\beta$. For any $\gamma, \delta \in \Gamma(\alpha)$, we have $\gamma^{g}, \delta^{g} \in \Gamma(\beta)$ and hence $\left(\gamma^{g}\right)^{x}=\delta^{g}$ holds for some $x \in G_{(B)}$ by the transitivity of $G_{(B)}$ on $\Gamma(\beta)$. Since $G_{(B)} \triangleleft G_{B}$ (Lemma 2.4(a)), we have $g x g^{-1} \in G_{(B)}$, and hence $\gamma^{g x g^{-1}}=\delta$ implies that $G_{(B)}$ is transitive on $\Gamma(\alpha)$. 
If $k=1$, then $\Gamma_{\mathcal{B}}(\alpha) \cap \Gamma_{\mathcal{B}}(\beta)=\emptyset$ for distinct $\alpha, \beta \in B$. Hence, if $g \in G_{B}$ fixes each block $C \in \Gamma_{\mathcal{B}}(B)$ setwise, then it also fixes each vertex in $B$. So we have $G_{[B]}<G_{(B)}$ in this case.

If $k \geq 2$, then by Remark 3.2, for any $C, D \in \Gamma_{\mathcal{B}}(B)$, either $\Gamma(C) \cap B=\Gamma(D) \cap B$ or $\Gamma(C) \cap \Gamma(D) \cap B=\emptyset$. Hence Theorem 3.3 applies, and the partition $\mathcal{B}^{*}$ defined therein is a nontrivial $G$-invariant partition of $V(\Gamma)$ and is a refinement of $\mathcal{B}$. The truth of the remaining statements in (b)(ii) follows from Theorem 3.3(b).

Now we suppose $G_{[B]} \not \leq G_{(B)}$. Then $B_{1}:=\alpha^{G_{[B]}}$ has cardinality at least two, where $\alpha \in B$. Hence it follows from Theorem 2.5 that the partition $\mathcal{B}_{1}$ (defined in (4) for $i=1$ ) is a nontrivial $G$-invariant partition of $V(\Gamma)$ and is a refinement of $\mathcal{B}$, and that the parameters $v_{1}, s_{1}, r_{1}$ with respect to $\mathcal{B}_{1}$ have the required properties.

For minimal nontrivial $G$-invariant partitions, we have the following result.

Theorem 4.2 Suppose $\Gamma$ is a G-locally quasiprimitive graph, where $G \leq \operatorname{Aut}(\Gamma)$. Suppose further that $\mathcal{B}$ is a minimal nontrivial $G$-invariant partition of $V(\Gamma)$. Then one of the following (a)-(c) holds.

(a) $G_{[B]}=G_{(B)}$ and $G$ is faithful on $\mathcal{B}$;

(b) $G_{[B]}<G_{(B)}$ and $k=1$;

(c) $\Gamma$ is a multicover of $\Gamma_{\mathcal{B}}$.

Moreover, if $\Gamma_{\mathcal{B}}$ is a complete graph, then the occurrence of (a) implies $G_{[B]}=G_{(B)}=1$; if $G_{[B]} \not \pm G_{(B)}$, then the occurrence of (c) implies that $G_{[B]}$ is transitive on $B$.

Proof: In the case where $G_{(B)}=G_{[B]}, G$ is faithful on $\mathcal{B}$ by Corollary 2.8(a). Suppose $G_{(B)} \neq G_{[B]}$. Then either $G_{(B)} \not G_{[B]}$ or $G_{[B]} \not \leq G_{(B)}$. In the former case, Theorem 4.1(b) applies. If (i) in Theorem 4.1(b) occurs, then we have $k=1$ and $G_{[B]}<G_{(B)}$, and hence (b) above occurs. If (ii) in Theorem 4.1(b) occurs, then by the minimality of $\mathcal{B}$, the partition $\mathcal{B}^{*}$ therein must coincide with $\mathcal{B}$; hence $\Gamma$ is a multicover of $\Gamma_{\mathcal{B}}$ and (c) holds. In the latter case where $G_{[B]} \not \leq G_{(B)}$, by Corollary 2.8 and the minimality of $\mathcal{B}$, we know that $\Gamma$ is a multicover of $\Gamma_{\mathcal{B}}$ (hence (c) above occurs), and moreover $G_{[B]}$ is transitive on $B$.

Now suppose that $\Gamma_{\mathcal{B}}$ is a complete graph, and that case (a) occurs. Then $G_{[B]}$ is the kernel of the action of $G$ on $\mathcal{B}$ and hence $G_{[B]}=G_{(B)} \triangleleft G$. This implies that $G_{(B)}=$ $g^{-1} G_{(B)} g=G_{\left(B^{g}\right)}$ for any $g \in G$. Since $B^{g}$ runs over all blocks of $\mathcal{B}$ when $g$ runs over $G$, this means that $G_{(B)}$ fixes each vertex of $\Gamma$, and hence by the faithfulness of $G$ on $V(\Gamma)$ we get $G_{[B]}=G_{(B)}=1$.

Recall that $G_{[\alpha]}$ is the subgroup of $G_{\alpha}$ fixing setwise each block $B \in \Gamma_{\mathcal{B}}(\alpha)$. So $G_{[\alpha]}$ induces an action on $\Gamma(\alpha) \cap B$. It may happen (see Lemma 4.4 below) that $G_{[\alpha]}$ is transitive on $\Gamma(\alpha) \cap B$, that is, $\Gamma(\alpha) \cap B$ is a $\left(G_{[\alpha]}\right)$-orbit on $\Gamma(\alpha)$. In this case we have the following theorem, which is a counterpart of [3, Lemma 3.1(b)].

Theorem 4.3 Suppose $\Gamma$ is a G-locally quasiprimitive graph admitting a nontrivial $G$ invariant partition $\mathcal{B}$. Suppose further that $G_{[\alpha]}$ is transitive on $\Gamma(\alpha) \cap B$, for some $\alpha \in V(\Gamma)$ and $B \in \Gamma_{\mathcal{B}}(\alpha)$. Then either 
(a) $\Gamma[B, C] \cong k \cdot K_{2}$ is a matching of $k$ edges, for adjacent blocks $B, C$ of $\mathcal{B}$; or

(b) $\Gamma$ is a bipartite graph with each part of the bipartition of a connected component contained in some block of $\mathcal{B}$, and $r=1$.

Proof: We first show that our assumption on $G_{[\alpha]}$ implies that $G_{[\alpha]}$ is transitive on $\Gamma(\alpha) \cap C$ for each $C \in \Gamma_{\mathcal{B}}(\alpha)$. In fact, since $B, C \in \Gamma_{\mathcal{B}}(\alpha), \alpha$ is adjacent to a vertex $\beta$ in $B$ and a vertex $\gamma$ in $C$. So there exists $g \in G_{\alpha}$ such that $\gamma^{g}=\beta$, and hence $C^{g}=B$. Now for any $\delta, \varepsilon \in \Gamma(\alpha) \cap C$, we have $\delta^{g}, \varepsilon^{g} \in \Gamma(\alpha) \cap B$ and hence, by our assumption that $G_{[\alpha]}$ is transitive on $\Gamma(\alpha) \cap B,\left(\delta^{g}\right)^{x}=\varepsilon^{g}$ holds for some $x \in G_{[\alpha]}$. Since $G_{[\alpha]} \unlhd G_{\alpha}$ (Lemma 2.4(c)), we have $g x g^{-1} \in G_{[\alpha]}$ and so $\delta^{g x g^{-1}}=\varepsilon$ implies that $G_{[\alpha]}$ is transitive on $\Gamma(\alpha) \cap C$. Thus, if $G_{[\alpha]}^{\Gamma(\alpha)}=1$, then we have $|\Gamma(\alpha) \cap C|=1$. That is, $\Gamma[B, C]$ is a matching for adjacent blocks $B, C$ of $\mathcal{B}$, and hence the statement in (a) holds.

In the following we suppose that $G_{[\alpha]}^{\Gamma(\alpha)} \neq 1$. Then, since $G_{[\alpha]}^{\Gamma(\alpha)} \unlhd G_{\alpha}^{\Gamma(\alpha)}$ by Lemma 2.4(c) and since $\Gamma$ is $G$-locally quasiprimitive by our assumption, $G_{[\alpha]}$ must be transitive on $\Gamma(\alpha)$. However, $G_{[\alpha]}$ fixes $\Gamma(\alpha) \cap C$ setwise for each $C \in \Gamma_{\mathcal{B}}(\alpha)$. So we must have $r=\left|\Gamma_{\mathcal{B}}(\alpha)\right|=1$ and hence $\Gamma(\alpha) \subseteq C$ for some $C$. Let $B$ be the block of $\mathcal{B}$ containing $\alpha$. Then, since $G$ is transitive on arcs of $\Gamma$, for any $\beta \in \Gamma(\alpha)$ there exists an element of $G$ which interchanges $\alpha$ and $\beta$ and hence interchanges $B$ and $C$. Hence $\Gamma(\alpha) \subseteq C$ implies $\Gamma(\beta) \subseteq B$. Similarly, $\Gamma(\beta) \subseteq B$ implies $\Gamma(\gamma) \subseteq C$ for any $\gamma \in \Gamma(\beta)$. Continuing this process, one can see that $\Gamma[B, C]$ consists of connected components of $\Gamma$, and hence each such component is a bipartite graph with the two parts of the bipartition contained in $B, C$, respectively. Therefore, $\Gamma$ is a bipartite graph.

The following lemma shows that $G_{[\alpha]}$ is transitive on $\Gamma(\alpha) \cap B$ for each $B \in \Gamma_{\mathcal{B}}(\alpha)$ provided that $G_{\alpha}$ is regular on $\Gamma_{\mathcal{B}}(\alpha)$. This will be used in the proof of Theorem 4.5 below.

Lemma 4.4 Suppose $\Gamma$ is a $G$-symmetric graph admitting a nontrivial $G$-invariant partition $\mathcal{B}$. If $G_{\alpha}$ is regular on $\Gamma_{\mathcal{B}}(\alpha)$, for some $\alpha \in V(\Gamma)$, then $G_{[\alpha]}$ is transitive on $\Gamma(\alpha) \cap B$ for each $B \in \Gamma_{\mathcal{B}}(\alpha)$.

Proof: For any $B \in \Gamma_{\mathcal{B}}(\alpha)$ and $\beta, \gamma \in \Gamma(\alpha) \cap B$, by the $G$-symmetry of $\Gamma$ there exists $x \in G_{\alpha}$ such that $\beta^{x}=\gamma$, and hence $x$ fixes $B$ setwise. Since by our assumption $G_{\alpha}$ acts regularly on $\Gamma_{\mathcal{B}}(\alpha)$, this implies that $C^{x}=C$ for all $C \in \Gamma_{\mathcal{B}}(\alpha)$, and hence $x \in G_{[\alpha]}$. Thus, any vertex $\beta$ in $\Gamma(\alpha) \cap B$ can be mapped to any other vertex $\gamma$ in $\Gamma(\alpha) \cap B$ by an element of $G_{[\alpha]}$. In other words, $G_{[\alpha]}$ is transitive on $\Gamma(\alpha) \cap B$.

We conclude this paper by proving the following result. A $G$-symmetric graph $\Gamma$ is said to be $(G, 1)$-arc regular if, in its induced action, $G$ is regular on $\operatorname{Arc}(\Gamma)$.

Theorem 4.5 Suppose $\Gamma$ is a connected, non-bipartite, G-locally quasiprimitive graph admitting a nontrivial $G$-invariant partition $\mathcal{B}$, where $G \leq \operatorname{Aut}(\Gamma)$. Suppose further that $G_{\alpha}$ is regular on $\Gamma_{\mathcal{B}}(\alpha)$ for $\alpha \in V(\Gamma)$. Then $\Gamma$ is $(G, 1)$-arc regular and $\Gamma[B, C] \cong k \cdot K_{2}$ for adjacent blocks $B, C$ of $\mathcal{B}$. 
Proof: Since $G_{\alpha}$ is regular on $\Gamma_{\mathcal{B}}(\alpha)$, by Lemma $4.4, G_{[\alpha]}$ is transitive on $\Gamma(\alpha) \cap B$ for $B \in \Gamma_{\mathcal{B}}(\alpha)$. Thus, since $\Gamma$ is non-bipartite, we have $\Gamma[B, C] \cong k \cdot K_{2}$ by Theorem 4.3. Consequently, the actions of $G_{\alpha}$ on $\Gamma_{\mathcal{B}}(\alpha)$ and $\Gamma(\alpha)$ are permutationally equivalent. So $G_{\alpha}$ is regular on $\Gamma(\alpha)$ as well. This together with the connectedness of $\Gamma$ implies that $\Gamma$ is $(G, 1)$-arc regular, as we show in the following.

Let $\Gamma(i, \alpha)$ denote the set of vertices of $\Gamma$ with distance no more than $i$ from $\alpha$. For any $\beta \in \Gamma(\alpha)$, since $G_{\alpha}$ is regular on $\Gamma(\alpha), G_{\alpha \beta}$ fixes each vertex in $\Gamma(\alpha) \cup\{\alpha, \beta\}$. Similarly, $G_{\beta \alpha}=G_{\alpha \beta}$ fixes each vertex in $\Gamma(\beta) \cup\{\alpha, \beta\}$. So $G_{\alpha \beta}$ fixes each vertex in $\Gamma(\alpha) \cup \Gamma(\beta) \cup\{\alpha, \beta\}$. Thus, for any vertex $\gamma \in \Gamma(\beta) \backslash\{\alpha\}$, we have $G_{\alpha \beta}=G_{\alpha \beta \gamma}$. Similarly, $G_{\gamma \beta}=G_{\alpha \beta \gamma}$ and so $G_{\alpha \beta}=G_{\gamma \beta}$. Repeating the argument above for the adjacent vertices $\gamma, \beta$, we know that $G_{\alpha \beta}\left(=G_{\gamma \beta}\right)$ fixes each vertex in $\Gamma(\gamma)$. Similarly, $G_{\alpha \beta}$ fixes each vertex in $\Gamma(\delta)$ for any $\delta \in \Gamma(\alpha) \backslash\{\beta\}$. Therefore, $G_{\alpha \beta}$ fixes each vertex in $\Gamma(2, \alpha) \cup \Gamma(2, \beta)$. Inductively, one can show that $G_{\alpha \beta}$ fixes each vertex in $\Gamma(i, \alpha) \cup \Gamma(i, \beta)$ for any $i \geq 1$. Since $\Gamma$ is connected, this implies that $G_{\alpha \beta}$ fixes each vertex of $\Gamma$. But $G \leq \operatorname{Aut}(\Gamma)$ is faithful on $V(\Gamma)$, so we have $G_{\alpha \beta}=1$ and $G$ is regular on the $\operatorname{arcs}$ of $\Gamma$.

\section{References}

1. N.L. Biggs, Algebraic Graph Theory, 2nd edn., Cambridge University Press, Cambridge, 1993.

2. J.D. Dixon and B. Morttimer, Permutation Groups, Springer, New York, 1996.

3. A. Gardiner and C.E. Praeger, "A geometrical approach to imprimitive graphs," Proc. London Math. Soc.(3) 71 (1995), 524-546.

4. A. Gardiner and C.E. Praeger, Symmetric graphs with complete quotients, preprint, University of Western Australia.

5. M.A. Iranmanesh, C.E. Praeger, and S. Zhou, "Finite symmetric graphs with two-arc transitive quotients," $J$. Combin. Theory (Series B) 94 (2005), 79-99.

6. C.H. Li, C.E. Praeger, A. Venkatesh, and S. Zhou, "Finite locally quasiprimitive graphs," Discrete Math. 246 (2002), 197-218.

7. C.H. Li, C.E. Praeger, and S. Zhou, "A class of finite symmetric graphs with 2-arc transitive quotients," Math. Proc. Camb. Phil. Soc. 129 (2000), 19-34.

8. C.E. Praeger, "Imprimitive symmetric graphs," Ars Combin. 19A (1985), 149-163.

9. C.E. Praeger, "On automorphism groups of imprimitive symmetric graphs," Ars Combin. 23A (1987), 207224.

10. C.E. Praeger, "Finite transitive permutation groups and finite vertex transitive graphs," in Graph Symmetry G. Hahn and G. Sabidussi (Eds.), (Montreal, 1996, NATO Adv. Sci. Inst. Ser. C, Math. Phys. Sci., 497), Kluwer Academic Publishing, Dordrecht, 1997, pp. 277-318.

11. C.E. Praeger, "Finite quasiprimitive graphs, surveys in combinatorics," London Math. Soc. Lecture Note Series 24 (1997), 65-85.

12. S. Zhou, "Imprimitive symmetric graphs, 3-arc graphs and 1-designs," Discrete Math. 244 (2002), 521-537.

13. S. Zhou, "Constructing a class of symmetric graphs," European J. Combin. 23 (2002), 741-760.

14. S. Zhou, "Symmetric graphs and flag graphs," Monatshefte für Mathematik 139 (2003), 69-81.

15. S. Zhou, "Almost covers of 2-arc transitive graphs," Combin. 24 (2004), 731-745. 
